Pacific Journal of Mathematics

THE DENSITY TOPOLOGY 


\title{
THE DENSITY TOPOLOGY
}

\author{
Franklin D. TALL
}

The density topology on the real line is a strengthening of the usual Euclidean topology which is intimately connected with the measure-theoretic structure of the reals. The purpose of this note is to treat the density topology from a modern topological viewpoint.

1. Introduction. A sprinkling of papers has been published by analysts on the density topology. We summarize the important results from the literature, and contribute some new ones. These mainly concern the characterization of certain subspaces, and consideration of cardinal invariants. Many of the topics we touch upon can be treated in more general measure-theoretic structures than the real line, but this does not appear to be particularly fruitful topologically. The organization of this paper is as follows: in $\$ 2$, results stated explicitly or inherent in the literature are given; in $\$ 3$, some new results are obtained, especially concerning various subspaces of $X$; in $\S 4$, the implications for $X$ of various set-theoretic hypotheses are examined.

\section{Definitions and results from the literature.}

Definition 2.1. A measurable set $E \subseteq \mathbf{R}$ has density $d$ at $x$ if

$$
\lim _{h \rightarrow 0} \frac{m(E \cap[x-h, x+h])}{2 h}
$$

exists and equals $d$. Different authors use slightly different definitions of density but they all agree in case $d=1$ and therefore determine the same topology. Denote by $\phi(E),\{x \in \mathbf{R}: d(x, E)=1\}$. Let $A \sim B$ mean $A \Delta B$ (the symmetric difference of $A$ and $B$ ) is a nullset (i.e. has measure zero).

THEOREM 2.2. (See e.g. [12].) Let $A$ be measurable. Then

(1) $\phi(A) \sim A$,

(2) if $A \sim B$, then $\phi(A)=\phi(B)$,

(3) $\phi(\varnothing)=\varnothing$ and $\phi(\mathbf{R})=\mathbf{R}$,

(4) $\phi(A \cap B)=\phi(A) \cap \phi(B)$,

(5) if $A \subseteq B$, then $\phi(A) \subseteq \phi(B)$. 
THEOREM 2.3. (See e.g. [2].) The family of all measurable sets $E$ such that $\phi(E) \supseteq E$ is a topology on $\mathbf{R}$, henceforth denoted by $\langle X, \mathscr{T}\rangle$ or just $X$ if confusion is unlikely. Clearly $\mathscr{T}$ is stronger than the usual topology.

THEOREM 2.4 [2]. $X$ is not normal; however $X$ is $T_{3 \frac{1}{2}}$, in fact given disjoint sets $F$ and $K$, one closed in $X$, the other in the Euclidean topology, there exists a continuous function $f: X \rightarrow[0,1]$ such that $f^{-1}(0)=F$ and $f^{-1}(1)=K$.

We shall later give a number of different proofs that $X$ is not normal. That $X$ is $T_{3^{\frac{1}{2}}}$ is proved in [2] using a consequence in [19] of the important Lusin-Menchoff Theorem :

Theorem 2.5. Suppose (in the Euclidean topology) that $F$ is closed, $B$ is Borel, $F \subseteq B$, and for every $x \in F, d(x, B)=1$. Then there is $a$ perfect set $P, F \subseteq P \subseteq B$, such that for every $x \in F, d(x, P)=1$.

THEOREM 2.6 [14].

(1) The Borel subsets of $X$ are precisely the measurable sets.

(2) Every Borel subset of $X$ is $a G_{\delta}$, in fact the intersection (or union) of an open $F_{\sigma}$ and $a$ closed $G_{\delta}$, namely any $E=$ $(E \cap \phi(E)) \cup(E-\phi(E))$, and similarly for $X-E$.

(3) Every regular open set is a Euclidean $F_{\sigma \delta}$.

THEOREM 2.7. The following conditions on a subset $Y$ of $X$ are equivalent:

(1) $Y$ is a nullset,

(2) $Y$ is nowhere dense,

(3) $Y$ is first category,

(4) $Y$ is closed discrete.

The equivalence of the first three is shown in [12], as well as that nowhere dense sets are closed, and hence closed discrete. If a closed discrete set were not a nullset, it would include a nonmeasurable set. But nonmeasurable sets are not closed. For a proof of 2.7 in a more general context, see [10].

THEOREM 2.8. $R O(X)$, the regular open algebra of $X$, is the reduced measure algebra $\mathscr{B}$ of measurable sets modulo nullsets. Moreover $\phi(A)=A$ if and only if $A$ is regular open.

The former is easily seen, since $R O(X)$ can be characterized as open sets modulo first category sets [4]. The latter is proved in [12]. 
THEOREM 2.9. $X$ satisfies the countable chain condition; indeed $X$ has property $(K)$ : every uncountable collection of open sets has an uncountable subcollection such that each pair meet.

This follows from thè corresponding result for measure algebras [6].

THEOREM 2.10. $X$ is connected [3]. However, there is a $T_{3_{2}^{\frac{1}{2}}}$ enlargement $\mathscr{S}$ of $\mathscr{T}$ which satisfies 2.7 inter alia, which is extremally disconnected and the Stone-Čech compactification of which coincides with the Stone space of $\mathscr{B}[14]$.

THEOREM 2.11. $X$ is neither separable nor first countable, but is hereditarily Baire (every subspace of $X$ is Baire).

This follows immediately from the facts that countable sets are nowhere dense and that first category sets are nowhere dense and closed.

THEOREM 2.12. The continuous real-valued functions of $X$ are of Baire class 1 and hence are continuous in the Euclidean topology except on a set of Euclidean first category [3].

The continuous real-valued functions on $X$ are in fact the "approximately continuous" functions. There is an extensive literature concerning these which does not however consider their topological aspects. See Goffman's papers for some references.

One may conclude various things about $X$ from the fact that it has a weaker separable metric topology. For example,

THEOREM 2.13. X X has a countable point-separating open cover and a regular $G_{\delta}$-diagonal.

Both properties are possessed by the Euclidean topology and are preserved by strengthening a topology.

$X$ possesses a variety of completeness properties. For definitions and proofs see [18].

THEOREM 2.14. $X$ is cocompact, strongly $\alpha$-favorable, and pseudocomplete.

3. New results. We now move on to some simple applications of the above results.

THEOREM 3.1. Every $Y \subseteq X$ is the union of a CCC (countable chain condition) set and a closed discrete set. 
Proof. $Y=(Y \cap$ int $\bar{Y}) \cup(Y \cap(\bar{Y}-$ int $\bar{Y}))$. The first term is $C C C$ since that property is inherited by open sets and by dense sets. The second is a subset of a nowhere dense set, and hence is closed discrete.

COROllary 3.2. Every discrete subspace of $X$ is closed.

Proof. Using the Theorem, we see that a discrete $Y$ is the union of a countable (hence closed) set and a closed set.

COROllary 3.3. $X$ is hereditarily subparacompact (every subspace has the property that open covers have $\sigma$-discrete closed refinements).

Proof. It suffices to prove $C C C$ subspaces $Y$ of $X$ are subparacompact. Given an open cover $\mathcal{O}$ of $Y$, refine it by an open cover $\mathcal{U}$ such that the closures of elements of $\mathcal{U}$ refine $\mathcal{O}$. Let $\left\{C_{n}\right\}_{n<\omega}$ be a maximal disjoint collection of open sets, each included in an element of $\mathcal{U}$. Then $Y-\cup_{n<\omega} C_{n}$ is nowhere dense in $Y$, hence in $X$, hence is closed discrete. A $\sigma$-discrete closed refinement of 0 can then be obtained by taking the points of $Y-\cup_{n<\omega} C_{n}$ as one level, and the $\overline{C_{n}}$ 's as the other levels.

DEFINITION 3.4. A space is $\kappa$-compact if every closed discrete subset of it has cardinality $<\kappa$. A space is $(\sigma-)$ metacompact if every open cover has a $(\sigma-)$ point-finite open refinement. A space is collectionwise Hausdorff if for each closed discrete subset $Y$ there exist pairwise disjoint open sets, each containing exactly one element of $Y$.

THEOREM 3.5.

(1) If $Y \subseteq X$ is $\boldsymbol{N}_{1}$-compact, $Y$ is hereditarily Lindelöf.

(2) If $Y \subseteq X$ is either collectionwise Hausdorff or $\sigma$-metacompact, $Y$ is the union of a hereditarily Lindelöf set and a closed discrete set.

(3) $X$ is neither collectionwise Hausdorff nor $\sigma$-metacompact.

Proof. It is easily seen that subparacompact $\boldsymbol{\aleph}_{1}$-compact spaces are Lindelöf. Since closed subsets of $X$ are $G_{\delta}$ 's, Lindelöf subsets are hereditarily Lindelöf. If $Y$ is collectionwise Hausdorff, it is the union of an $\boldsymbol{\aleph}_{1}$-compact set and a closed discrete set. In [17] it is shown that $C C C$ Baire $\sigma$-metacompact spaces are Lindelö, which completes the proof of the second part, and also shows $X$ is not $\sigma$-metacompact. $X$ is clearly not collectionwise Hausdorff.

THEOREM 3.6.

(1) Countably compact subsets of $X$ are finite.

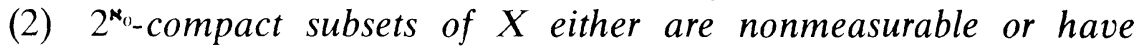
cardinality $<2^{\boldsymbol{N}_{0}}$. 
The first is because countable sets are closed discrete, the second because every measurable set of power $2^{\boldsymbol{\aleph}_{0}}$ includes a nullset of power $2^{\boldsymbol{\aleph}_{0}}$.

Our next theorem provides a proof of the nonnormality of $X$ using very little information about its measure-theoretic structure, in contrast to other proofs [2], [14].

THEOREM 3.7. Let $Y$ be a normal topological space such that $|R O(Y)|<2^{\kappa}$. Then $Y$ is $\kappa$-compact.

COROllary 3.8 .

(1) $X$ is not normal,

(2) if $Y \subseteq X$ is normal, $Y$ is the union of a $2^{\aleph_{0}-c o m p a c t ~ s e t ~ a n d ~ a ~}$ closed discrete set,

(3) if $2^{\boldsymbol{\aleph}_{0}}<2^{\boldsymbol{\aleph}_{1}}$ and $Y \subseteq X$ is normal, $Y$ is the union of a hereditarily Lindelöf set and a closed discrete set.

We first prove the Corollary. To see that $X$ is not normal, we note

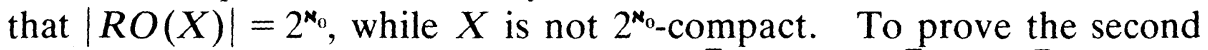
part, we note as before that $Y=(Y \cap$ int $\bar{Y}) \cup(Y \cap(\bar{Y}-$ int $\bar{Y}))$. Surely $R O$ (int $\bar{Y}) \subseteq R O(X)$. On the other hand, $Y$ is dense in int $\bar{Y}$, and it is easily verified that in general, for a regular space $Z, Y$ dense in $Z$ implies $|R O(Y)|=|R O(Z)|$. Thus $\mid R O(Y \cap$ int $\bar{Y}) \mid \leqq 2^{\aleph_{0}}$, so $Y \cap$ int $\bar{Y}$ is $2^{\boldsymbol{N}_{0}}$-compact, and if $2^{\boldsymbol{\kappa}_{0}}<2^{\boldsymbol{\kappa}_{1}}, \boldsymbol{N}_{1}$-compact and therefore hereditarily Lindelöf.

Theorem 3.7 provides a useful method for showing many spaces not to be normal. The idea is due to B. Śapirovskii [15]. To prove it, suppose $Y$ is not $\kappa$-compact but is normal. Let $Z$ be a closed discrete subspace of $Y$ of cardinality $\kappa$. Let $K$ be any subset of $Z$. By normality, there exists an open set $U_{K} \supseteq K$ such that $\bar{U}_{K} \cap(Z-K)=\varnothing$. Int $\bar{U}_{K}$ is regular open and includes $K$. It is not difficult to establish that $K \rightarrow$ int $\bar{U}_{K}$ is a one-one map from the power set of $Z$ into $R O(Y)$.

Another way of proving the nonnormality of $X$ is to again observe that $X$ is not $2^{\boldsymbol{N}_{0}}$-compact, and that there are only $2^{\boldsymbol{\kappa}_{0}}$ real-valued continuous functions on $X$, not enough to provide sufficiently many Urysohn functions. The fact that there are only $2^{\kappa_{0}}$ real-valued continuous functions on $X$ follows from the fact that they are all of Baire class 1 , i.e. limits of sequences of ordinary continuous functions.

The proof of nonnormality of $X$ in [2] yields further information: disjoint Euclidean-dense sets cannot be separated by a Urysohn function. In particular, the rationals and $\{r \sqrt{2}: r$ rational $\}$ cannot be. On the other hand, since $X$ is regular and countable sets are closed, it is not difficult to show that disjoint countable sets have disjoint open sets about them. I have been unable to determine whether disjoint closed sets, one of which is countable, have disjoint open sets about them. 
Analogous results to those for normality hold for countable paracompactness, thanks to the following result, which can be proved by an analysis of [1].

THEOREM 3.9. Let $Y$ be a countably paracompact space such that $|R O(Y)| \leqq \kappa$. Then $Y$ is $\kappa$-compact.

Corollary 3.10.

(1) $X$ is not countably paracompact,

(2) if $Y \subseteq X$ is countably paracompact, $Y$ is the union of a $2^{\boldsymbol{N}_{0}}$-compact set and a closed discrete set,

(3) if $2^{\aleph_{0}}=\aleph_{1}$ and $Y \subseteq X$ is countably paracompact, $Y$ is the union of a hereditarily Lindelöf set and a closed discrete set.

In view of the well-known analogies between measure and category [12], it is natural to ask whether there is a category analogue of the density topology. If this question is formulated precisely in a natural way, the answer is no.

THEOREM 3.11. There is no topology $\mathscr{S}$ on the real line such that $Y=\langle\mathbf{R}, \mathscr{S}\rangle$ has the following properties

(1) $Y$ is regular,

(2) $R O(Y)=\mathscr{B}^{\prime}$, the reduced Borel algebra of Euclidean Borel sets modulo Euclidean first category sets.

(3) if $U$ is Euclidean open and $N$ is Euclidean first category, then $U-N \in \mathscr{S}$.

Proof. We first recall some facts about Boolean algebras (see e.g. [4]). If $\mathscr{A}$ is a Boolean algebra, define a partial order on $P=\mathscr{A}-\{0\}$ by $a \leqq b$ if $a \wedge b=a$. A subset $D$ of $P$ is dense if for each $a \in P$ there is a $d \in D$ such that $d \leqq a$. For any space $Z$, the $\leqq$ in $R O(Z)$ is just inclusion. A $\pi$-base for a space $Z$ is a dense subset of the inclusion ordering on the nonempty open sets. Thus, if $Z$ is regular, there are $\pi$-bases of regular open sets and these are exactly the dense subsets of $R O(Z)$ (more precisely $R O(Z)-\{0\}$ ). Finally, we recall from [4] that $\mathscr{B}^{\prime}$ has a countable dense subset and is atomless (no minimal elements in the partial order).

Returning to the Theorem, we see that $Y$ has a countable $\pi$-base and is therefore separable. But this contradicts (3).

(3) may be replaced by

$\left(3^{\prime}\right)$ every first category subset of $Y$ is nowhere dense in $Y$. The point is that, since $\mathscr{B}^{\prime}$ is atomless, $Y$ has no isolated points, so countable sets are first category. 
4. Set theory. There has lately been considerable interest in the question of the existence of hereditarily Lindelöf nonseparable regular spaces [13]. A Souslin space is one, and Hajnal and Juhász [5] construct one, assuming the continuum hypothesis. A much simpler example than theirs, also assuming the continuum hypothesis, is due to H. E. White [18], who, unaware of the problem, failed to make the (trivial) observation that the hereditarily Lindelöf subspace of the density topology he constructed is nonseparable. We shall elaborate on the example here.

A Sierpinski set is a set of reals which has countable intersection with every nullset.

LEMMA 4.1 [16]. The continuum hypothesis implies the existence of an uncountable Sierpinski set.

THEOREM 4.2. $Y \subseteq X$ is hereditarily Lindelöf if and only if $Y$ is a Sierpinski set.

COROLlaRy 4.3. The continuum hypothesis implies the existence of a hereditarily Lindelöf, nonseparable, regular Baire space.

The corollary is immediate. To prove the theorem, observe that if $Y$ is hereditarily Lindelöf, then every closed discrete subspace is countable. Conversely, if $Y$ is a Sierpinski set, then every nowhere dense subset of $Y$ is countable. Therefore, by 3.1, $Y$ is the union of a $C C C$ set $Y_{1}$ and a countable set $Y_{2}$.

LEMMA 4.4. A space is hereditarily Lindelö if and only if it is CCC and nowhere dense subsets are Lindelöf.

The proof is left to the reader. $Y$ then is the union of a hereditarily Lindelöf set and a countable set, and so is hereditarily Lindelöf.

It is set-theoretic folklore that there are models of set theory in which $2^{\boldsymbol{N}_{0}}>\boldsymbol{N}_{1}$ and there are uncountable Sierpinski sets, and that there are models of set theory in which there are no uncountable Sierpinski sets. Thus

THEOREM 4.5. It is consistent with the axioms of set theory that $2^{\boldsymbol{N}_{0}}>\boldsymbol{N}_{1}$ and there exists a hereditarily Lindelöf nonseparable regular space.

THEOREM 4.6. It is consistent with the axioms of set theory that the only hereditarily Lindelof subspaces of $X$ are the countable ones. 
It is perhaps of interest that an uncountable Sierpinski set may be used to construct a hereditarily Lindelöf nonseparable subspace of $\beta N-N$. First we note that in Scheinberg's extremally disconnected strengthening of the density topology [14], uncountable Sierpinski sets are again hereditarily Lindelöf nonseparable. Scheinberg's space has the Stone space of the reduced measure algebra for its Stone-Cech compactification. Kunen [8] has proven that this Stone space is embedded in $\beta N-N$.

We next prove some more consistency results.

Definition 4.7. A space is metalindelöf if every open cover has a point-countable open refinement. A space has caliber $\boldsymbol{N}_{1}$ if every point-countable open cover is countable.

THEOREM 4.8. The assertions that $X$ is not metalindelöf and that $X$ has caliber $\boldsymbol{N}_{1}$ are consistent with and independent of the axioms of set theory.

Proofs. Consistency is provided by Martin's Axiom plus $2^{\boldsymbol{\aleph}_{0}}>\boldsymbol{N}_{1}$, independence by the continuum hypothesis. Martin's Axiom plus $2^{\boldsymbol{N}_{0}}>\boldsymbol{N}_{1}$ implies the union of $\boldsymbol{N}_{1}$ nullsets is a nullset [11]. A CCC space in which the union of $\boldsymbol{N}_{1}$ nowhere dense sets is first category, has caliber $\boldsymbol{N}_{1}$ [17]. $X$ is not Lindelöf, so if it has caliber $\boldsymbol{N}_{1}$ it is not metalindelöf. By arguing as in 3.5, one can in fact prove that if the union of $\boldsymbol{N}_{1}$ nullsets is a nullset then every metalindelöf subset of $X$ is the union of a hereditarily Lindelöf set and a closed discrete set.

One can prove that the continuum hypothesis implies $X$ does not have caliber $\boldsymbol{N}_{1}$ by the same methods used to establish this result for the Stone space of the reduced measure algebra in [9]. However it will also follow immediately once we get $X$ metalindelöf, which is a consequence of

THEOREM 4.9. If every set of power $<2^{\boldsymbol{\aleph}_{0}}$ has measure zero, then every open cover of $X$ has a point $-<2^{\aleph_{0}}$ open refinement.

Proof. Let $X=\left\{x_{\alpha}\right\}_{\alpha<2^{\boldsymbol{\alpha}_{0}}}$. Let $\mathcal{U}=\left\{U_{\beta}\right\}_{\beta<2^{\boldsymbol{\alpha}_{0}}}$ be an open cover of $X$. For each $\alpha$, let $f(\alpha)$ be the least $\beta$ such that $x_{\alpha} \in U_{\beta}$. Let

$$
V_{\beta}=U_{\beta}-\left\{x_{\alpha}: \alpha<\beta \text { and } f(\alpha) \neq \beta\right\}
$$

Then $\mathscr{V}=\left\{V_{\beta}\right\}_{\beta<2^{\boldsymbol{\kappa}_{0}}}$ is the desired refinement.

We next consider the cardinal invariants of $X$. For definitions, see [7]. Since $X$ has a closed discrete subspace of cardinality $2^{\boldsymbol{\aleph}_{0}}$, we have 
THEOREM 4.10. The spread, weight, and Lindelof number of $X$ are all $2^{\mathrm{N}_{0}}$.

Since countable sets are closed, it is easy to see that

THEOREM 4.11. The density, tightness, $\pi$-weight, and character of $X$ are all uncountable but $\leqq 2^{\aleph_{0}}$.

Similarly,

THEOREM 4.12. Suppose every set of reals of power $<2^{\mathrm{N}_{0}}$ has meas ure zero. Then the density, tightness, $\pi$-weight, and character are all $2^{\boldsymbol{\kappa}_{0}}$.

Thus Martin's Axiom or the continuum hypothesis settles the question. For any space $Y$, it is known that $d(Y) \leqq \pi(Y), t(Y) \leqq \chi(Y)$, $\pi(Y) \leqq d(Y) \cdot \chi(Y)$. We shall show that for the density topology, $d(X) \leqq t(X)$ and hence $\pi(X) \leqq \chi(X)$. This follows from measuretheoretic characterizations of the density and tightness.

THEOREM 4.13.

(1) $d(X)$ is the least cardinal $\kappa$ such that there is a subset of $X$ of cardinality $\kappa$ with outer measure 1.

(2) $t(X)$ is the least cardinal $\kappa$ such that every nonnullset includes a subset of power $\leqq \kappa$ with the same outer measure.

Corollary $4.14 . \quad d(X) \leqq t(X), \pi(X) \leqq \chi(X)$.

Proof. It is clear that $d$ and $t$ are respectively at least as big as the cardinals defined on the right. A set of outer measure 1 is clearly dense. Similarly, if every nonnullset $Y$ includes a subset $Z$ of power $\leqq$ $\kappa$ with the same outer measure, then if $x \in \bar{Y}$, either $x \in Y$ or $x \in$ $\bar{Z}$. Hence $t(x, Y) \leqq \kappa$.

The next few words are directed to an audience versed in set theory. In the model obtained by adjoining $\boldsymbol{N}_{2}$ random reals to a model of the continuum hypothesis, it is well-known that $2^{\boldsymbol{N}_{0}}=\boldsymbol{N}_{2}$ and that the reals of the ground model have outer measure 1 in the extension. $K$. Kunen pointed out to the author that in fact in this model every nonnullset includes a subset of power $\boldsymbol{N}_{1}$ with the same outer measure. On the other hand, he noted that by adjoining $\boldsymbol{N}_{1}$ random reals to a model of Martin's Axiom plus $2^{\boldsymbol{N}_{0}}>\boldsymbol{N}_{1}$, one obtains a model in which there is a set of power $\boldsymbol{N}_{1}$ with outer measure 1 , and yet there is a set of power $>\boldsymbol{N}_{1}$ (namely the reals of the ground model) which does not include any subset of power $\boldsymbol{N}_{1}$ with the same outer measure. Thus

THEOREM 4.15. It is consistent that $d(X)=t(X)=\boldsymbol{\aleph}_{1}<2^{\boldsymbol{\aleph}_{0}}$. 
THEOREM 4.16. It is consistent that $d(X)<t(X)$.

By the remarks at the end of $\$ 3$, the question of whether the $\pi$-weight of $X$ can be less than continuum translates into asking whether there can be a dense subset of the reduced measure algebra of cardinality less than continuum. In response to the author's question, R. M. Solovay and $\mathrm{K}$. Kunen both proved that in the model obtained by adjoining $\boldsymbol{N}_{2}$ Sacks reals to a model of the continuum hypothesis, there is such a dense set of power $\boldsymbol{N}_{1}$. Kunen's proof also established that in that model the character of $X$ is $\boldsymbol{N}_{1}$. Thus

\section{THEOREM 4.17. It is consistent that $\pi(X)=\chi(X)=\boldsymbol{\aleph}_{1}<2^{\boldsymbol{\kappa}_{0}}$.}

In conclusion, I should like to thank the referee for his helpful comments.

\section{REFERENCES}

1. W. Fleissner, $\mathrm{CH}$ implies that countably paracompact, separable Moore spaces are normal, preprint.

2. C. Goffman, C. J. Neugebauer, and T. Nishiura, Density topology and approximate continuity, Duke Math. J., 28 (1961), 497-506.

3. C. Goffman and D. Waterman, Approximately continuous transformations, Proc. Amer. Math. Soc., 12 (1961), 116-121.

4. P. R. Halmos, Lectures on Boolean Algebras, Van Nostrand, Princeton, 1963.

5. A. Hajnal and I. Juhász, On hereditarily $\alpha$-Lindelöf and $\alpha$-separable spaces, II, Fund. Math., 81 (1974), 147-158.

6. A. Horn and A. Tarski, Measures in boolean algebras, Trans. Amer. Math. Soc., 64 (1948), 467-497.

7. I. Juhász, Cardinal functions in topology, Mathematical Centre, Amsterdam, 1971.

8. K. Kunen, Some points in $\beta N$, preprint.

9. K. Kunen and F. D. Tall, Between Martin's Axiom and Souslin's Hypothesis, preprint.

10. N. F. G. Martin, A topology for certain measure spaces, Proc. Amer. Math. Soc., 20 (1964), 1-18.

11. D. Martin and R. M. Solovay, Internal Cohen extensions, Ann. Math. Logic, 2 (1970), 143-178.

12. J. C. Oxtoby, Measure and category, Springer-Verlag, New York, 1971.

13. M. E. Rudin, Lectures on set-theoretic topology, Regional conf. series in math. 23, Amer. Math.

Soc., Providence, 1975.

14. S. Scheinberg, Topologies which generate a complete measure algebra, Adv. in Math., 7 (1971), 231-239.

15. B. Šapirovskiř, On separability and metrizability of spaces with Souslin's condition, Soviet Math. Dokl., 13 (1975), 1633-1638.

16. W. Sierpiński, Hypothèse du continu, Chelsea, New York, 1956.

17. F. D. Tall, The countable chain condition versus separability - applications of Martin's Axiom, Gen. Top. Appl., 4 (1974), 315-339.

18. H. E. White, Topological spaces in which Blumberg's theorem holds, Proc. Amer. Math. Soc., 44 (1974), 454-462.

19. Z. Zahorski, Sur la première dérivée, Trans. Amer. Math. Soc., 69 (1950), 1-54.

Received April 1, 1975 and in revised form October 1, 1975. The preparation of this paper was assisted by Grant A-7354 of the National Research Council of Canada. 



\section{Pacific Journal of Mathematics}

\section{Vol. 62, No. $1 \quad$ January, 1976}

Mieczyslaw Altman, Contractor directions, directional contractors and

directional contractions for solving equations . .................. 1

Michael Peter Anderson, Subgroups of finite index in profinite groups .........

Zvi Arad, Abelian and nilpotent subgroups of maximal order of groups of odd order

John David Baildon and Ruth Silverman, On starshaped sets and Helly-type theorems ..........................................

John W. Baker and R. C. Lacher, Some mappings which do not admit an

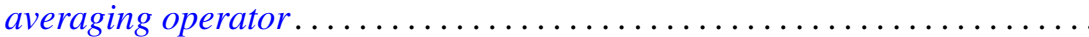

Joseph Barback, Composite numbers and prime regressive isols . . . . . . . . . .

David M. Boyd, Composition operators on $H^{p}(A) \ldots \ldots \ldots \ldots \ldots \ldots \ldots$

Maurice Chacron, Co-radical extension of PI rings . . . . . . . . . . . . .

Fred D. Crary, Some new engulfing theorems . . . . . . . . . . . . . . .

Victor Dannon and Dany Leviatan, A representation theorem for convolution transform with determining function in $L^{p} \ldots \ldots \ldots \ldots \ldots \ldots \ldots \ldots \ldots \ldots \ldots \ldots \ldots \ldots$

Mahlon M. Day, Lumpy subsets in left-amenable locally compact

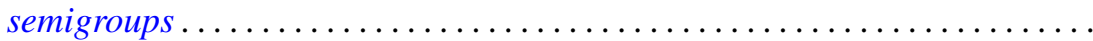

Michael A. Gauger, Some remarks on the center of the universal enveloping algebra of a classical simple Lie algebra . .

David K. Haley, Equational compactness and compact topologies in rings

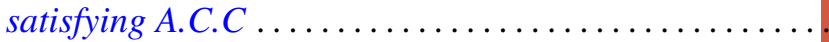

Raymond Heitmann, Generating ideals in Prüfer domains .

Gerald Norman Hile, Entire solutions of linear elliptic equations with

Laplacian principal part. .

Richard Oscar Hill, Moore-Postnikov towers for fibrations in which $\pi_{1}$ (fiber) is non-abelian

John Rast Hubbard, Approximation of compact homogeneous maps . .

Russell L. Merris, Relations among generalized matrix functions . .

V. S. Ramamurthi and Edgar Andrews Rutter, On cotorsion radicals ...

Ralph Tyrrell Rockafellar and Roger Jean-Baptiste Robert Wets, Stochastic

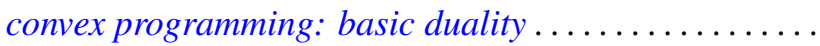

Alban J. Roques, Local evolution systems in general Banach spaces ..

I. Bert Russak, An indirect sufficiency proof for problems with bounded state variables.

Richard Alexander Sanerib, Jr., Ultrafilters and the basis property. .

H. A. Seid, The decomposition of multiplication operators on $L_{p}$-spaces . .

Franklin D. Tall, The density topology .................. 